TITLE:

\title{
Two-step Monte Carlo sensitivity analysis of alpha- and gamma- eigenvalues with the differential operator sampling method
}

\section{AUTHOR(S):}

Yamamoto, Toshihiro; Sakamoto, Hiroki

\section{CITATION:}

Yamamoto, Toshihiro ...[et al]. Two-step Monte Carlo sensitivity analysis of alpha- and gamma-eigenvalues with the differential operator sampling method. Annals of Nuclear Energy 2019, 133: 100-109

\section{ISSUE DATE:}

2019-11

URL:

http://hdl.handle.net/2433/241540

\section{RIGHT:}

(c) 2019. This manuscript version is made available under the CC-BY-NC-ND 4.0 license

http://creativecommons.org/licenses/by-nc-nd/4.0/:; The full-text file will be made open to the public on 1 November 2021 in accordance with publisher's 'Terms and Conditions for Self-Archiving'.; この論文は出版社版でありません。引 用の際には出版社版をご確認ご利用ください。; This is not the published version. Please cite only the published version. 
Two-step Monte Carlo sensitivity analysis of alpha- and gamma-eigenvalues with the differential operator sampling method \\ ${ }^{b}$ Radiation Dose Analysis and Evaluation Network, 4-13-14, Kokubunji-shi, Tokyo, 185-0001, \\ Japan
}

\begin{abstract}
A new Monte Carlo method is developed to calculate the sensitivity coefficients of the $\alpha$-eigenvalue (the time decay constant) and the $\gamma$-eigenvalue (the spatial decay constant in an exponential experiment) with respect to nuclear data. A method that was previously developed for the sensitivity analyses of the $\alpha$-eigenvalue, which is not based on the normal $k$ - $\alpha$ algorithm, is not applicable to the $\gamma$-eigenvalue due to its inability to obtain a converged source distribution. Then, a two-step method in which two sensitivity coefficients are separately calculated using the $k$ - $\alpha$ or $k-\gamma$ algorithm and the differential operator sampling method is newly developed. The sensitivity coefficient of the $\alpha$ - or $\gamma$-eigenvalue is represented by the ratio of the two sensitivity coefficients. Some numerical tests for three-energy group problems are performed using the new method. The sensitivity coefficients that are obtained by the new method are verified by comparing them to the solutions of deterministic transport calculations or to the approximate results that are obtained from the direct perturbations of the cross-sections.
\end{abstract}

Keywords: alpha-eigenvalue; gamma-eigenvalue, Monte Carlo; sensitivity analysis; differential operator

\title{
1. Introduction
}

These days, the development of Monte Carlo methods for sensitivity and uncertainty (S/U) analysis with respect to nuclear data has been intensively conducted. The capability of the

\footnotetext{
* Corresponding author. Tel:+81 72451 2414; fax:+81 724512658

E-mail address: toshihiro.yamamoto223@gmail.com (T. Yamamoto)
} 
sensitivity analysis of the $k_{\text {eff }}$-eigenvalue or neutron general responses (e.g., the capture to fission ratio) are now installed in many production-level Monte Carlo calculation codes, such as SCALE (Rearden, 2004; Perfetti, 2012; Perfetti and Rearden, 2016), MCNP (Kiedrowski et al., 2011; Kiedrowski and Brown, 2013), SERPENT (Aufiero et al., 2015), TRIPOLI (Terranova et al., 2018), MORET (Jinaphanh et al., 2016), McCARD (Shim and Kim, 2011), and RMC (Qiu et al., 2015; Qiu et al., 2016a; Qiu et al., 2016b).

In addition to the sensitivity analyses of the $k_{\text {eff }}$-eigenvalue and neutron general responses, the sensitivity analysis of the $\alpha$-eigenvalue (the prompt neutron time decay constant) is also important for the purpose of retaining the accuracy of calculation predictions of subcritical systems because the $\alpha$-eigenvalue is closely related with the subcriticality. Recently, some deterministic methods for the sensitivity coefficients of the $\alpha$-eigenvalue that use the forward and adjoint fluxes in an $\alpha$-eigenvalue mode calculation were developed by Endo and Yamamoto (2018) and Favorite (2018). A new Monte Carlo method for the sensitivity coefficients of the $\alpha$-eigenvalue was developed by the authors of this paper (Yamamoto and Sakamoto, 2019). Unlike the conventional Monte Carlo methods for the $\alpha$-eigenvalue calculation that use the power iteration of fission sources (the " $k$ - $\alpha$ " iteration method hereafter) (Brockway et al., 1985; Zoia et al., 2014), the new method introduces the "time source" that is the fundamental-mode eigenfunction of the $\alpha$-eigenvalue mode transport equation (Shim et al., 2014; Shim et al., 2015). In the time source method, the $\alpha$ is truly the eigenvalue of the $\alpha$-mode transport equation. Although the time source method outperforms the conventional $k$ - $\alpha$ iteration method for deep subcritical systems in terms of its robustness in stability, it has several weaknesses. The normal Monte Carlo procedure can generate no time source in a void region. To circumvent this weakness, a virtual total cross-section is assigned in the void region, which is similar to the Woodcock delta tracking method (Woodcock, 1965). In the time source method, the fission neutrons and all their progenies that are generated in a cycle have to be followed within the cycle, just as with a fixed source problem. As a system gets closer to criticality, the computation time for a cycle becomes longer.

Another important eigenvalue whose sensitivity coefficient is analyzed in a subcritical system 
1 is the $\gamma$-eigenvalue. The $\gamma$-eigenvalue is the spatial decay constant of the neutron flux in a

2 subcritical system that has a uniform composition in one of three directions. The spatial decay constant is obtained from an exponential experiment by fitting the neutron flux distribution to an exponential function (Suzaki, 1991; Yamamoto et al., 2003). If the flux is fitted in a region far enough away from the neutron source in order to exclude the effect of higher harmonics, the decay constant is equal to the fundamental mode eigenvalue of the $\gamma$-eigenvalue mode transport equation. The $\gamma$-eigenvalue is an important indicator of the subcriticality. This indicator is useful for the criticality safety control of fresh or spent nuclear fuel assemblies (Suzaki et al., 1995). The $\gamma$-eigenvalue correlates to the subcriticality more strongly than the $\alpha$-eigenvalue, especially in a deep subcritical system where the $\alpha$-eigenvalue increases very little regardless of the increase in the subcriticality (Suzaki, 1991). A Monte Carlo algorithm for calculating the $\gamma$-eigenvalue, which solves the $\gamma$-eigenvalue mode transport equation, was developed by Yamamoto and Miyoshi (2003) and Yamamoto (2012). The algorithm is essentially the same as the $k$ - $\alpha$ iteration method. However, unlike the $\alpha$-eigenvalue, the algorithm for the time source method cannot be applied to the $\gamma$-eigenvalue due to a reason that will be described later. Thus, we still have to use the $k$ - $\alpha$-like iteration method for the sensitivity analysis of the $\gamma$-eigenvalue (in this case, it should be called the “ $k-\gamma$ ” iteration method).

The objective of this paper is to propose a novel two-step Monte Carlo method for sensitivity analysis that uses the $k-\alpha$ or $k-\gamma$ iteration method instead of the time source method. The proposed method is applicable to the sensitivity analyses of the $\alpha$ - and $\gamma$-eigenvalue. The new method is particularly the only way for the sensitivity coefficients of the $\gamma$-eigenvalue to be calculated. The theory and the numerical tests of the new method are presented in the sections that follow.

\section{Review of Monte Carlo $\alpha$ - and $\gamma$-eigenvalue calculation methods}

\section{$2.1 k$ - $\alpha$ method}

Before proceeding to the newly developed two-step method, the conventional Monte Carlo method for obtaining the $\alpha$ - and $\gamma$-eigenvalues are briefly reviewed.

The neutron transport equation to be solved for obtaining the $\alpha$-eigenvalue is 


$$
\boldsymbol{L} \phi_{p}(\boldsymbol{r}, \boldsymbol{\Omega}, E)-\frac{\alpha}{v(E)} \phi_{p}(\boldsymbol{r}, \boldsymbol{\Omega}, E)=\frac{1}{k_{p}} \boldsymbol{F}_{p} \phi_{p}(\boldsymbol{r}, \boldsymbol{\Omega}, E) .
$$

The operators in Eq. (1) are written as

$$
\begin{gathered}
\boldsymbol{L} \phi_{p}(\boldsymbol{r}, \boldsymbol{\Omega}, E)=\boldsymbol{\Omega} \cdot \nabla \phi_{p}(\boldsymbol{r}, \boldsymbol{\Omega}, E)+\Sigma_{t}(\boldsymbol{r}, E) \phi_{p}(\boldsymbol{r}, \boldsymbol{\Omega}, E) \\
-\int_{4 \pi} d \boldsymbol{\Omega}^{\prime} \int d E^{\prime} \Sigma_{s}\left(\boldsymbol{r}, \boldsymbol{\Omega}^{\prime} \rightarrow \boldsymbol{\Omega}, E^{\prime} \rightarrow E\right) \phi_{p}\left(\boldsymbol{r}, \boldsymbol{\Omega}^{\prime}, E^{\prime}\right)
\end{gathered}
$$

2 and

$$
\boldsymbol{F}_{\boldsymbol{p}} \phi_{p}(\boldsymbol{r}, \boldsymbol{\Omega}, E)=\frac{\chi_{p}(E)}{4 \pi} \int_{4 \pi} d \boldsymbol{\Omega}^{\prime} \int d E^{\prime} v_{p} \Sigma_{f}\left(\boldsymbol{r}, E^{\prime}\right) \phi_{p}\left(\boldsymbol{r}, \boldsymbol{\Omega}^{\prime}, E^{\prime}\right),
$$

3 where $\Sigma_{t}=$ the macroscopic total cross-section, $\Sigma_{s}=$ the macroscopic scattering cross-section, $4 \quad \Sigma_{f}=$ the macroscopic fission cross-section, $\chi_{p}=$ the prompt neutron spectrum, $v_{p}=$ the number 5 of prompt neutrons per fission, $\phi_{p}=$ the fundamental mode eigenfunction of the $\alpha$-eigenvalue 6 mode equation, $\boldsymbol{\Omega}=$ the neutron direction, and $v=$ the neutron velocity. Although $k_{p}$ in Eq. (1) is 7 supposed to be unity in the $\alpha$-eigenvalue mode equation, it is explicitly shown. A commonly 8 adopted method for obtaining the fundamental mode $\alpha$-eigenvalue is the $k$ - $\alpha$ method, in which the $9 k_{p}$-eigenvalue calculation is performed with an additional term containing the $\alpha$-eigenvalue (i.e., the second term on the left-hand side of Eq. (1)). In the method proposed by Yamamoto and Miyoshi (2003), the additional term is handled during the course of the random walk process by changing the particle weight as it flies the distance $s_{j}$ :

$$
W_{j+1}=W_{j} \cdot \exp \left(\frac{\alpha}{v_{j}(E)} s_{j}\right),
$$

where $s_{j}=$ the $j$ th flight path, $v_{j}(E)=$ the neutron velocity of the $j$ th flight path, and $W_{j}$ and $W_{j+1}=$ the particle weight before and after the $j$ th flight path, respectively. This technique is referred to as the "continuous absorption weighting" (CAW) in the field of optics (Hayakawa et al., 2014). Using Eq. (4), the additional term can be incorporated into the $\alpha$-eigenvalue mode calculation, even for a void region where no collision occurs. The Monte Carlo algorithm to solve Eq. (1) is almost the same as the one for the $k_{\text {eff }}$-eigenvalue calculations. Thus, the "source" of the $k-\alpha$ method is the fission source, and the true eigenvalue is $k_{p}$ instead of $\alpha$. The $\alpha$ is an adjustment parameter that makes $k_{p}$ unity. In each cycle of the power iteration, $k_{p}$ is calculated with an $\alpha$ value in the same manner as the $k_{\text {eff }}$-eigenvalue calculation. The $\alpha$ that is used for the next cycle is determined so that $k_{p}$ approaches unity as 


$$
\alpha_{m+1}=\alpha_{m}+c\left(1-k_{p, m}\right),
$$

where $m$ is the cycle number and $c$ is an arbitrary positive value. If the $c$ value is not large enough, the convergence is slow and the inter-cycle correlation of the $\alpha$ becomes strong. If this value is too large, the variances of the $\alpha$ and $k_{\text {eff }}$ become unnecessarily large. An appropriate $c$ value should be determined by trial and error by monitoring the convergence and the fluctuation of the $\alpha$ in each cycle.

\section{$2.2 k-\gamma$ method}

This section briefly describes the Monte Carlo algorithm of the $k-\gamma$ method. Suppose a subcritical system where the horizontal buckling in the $x$ - and $y$-directions is larger than the material buckling and that an external neutron source is located far enough away in the $z$-direction. If the subcritical system is homogeneous and infinitely long in the $z$-direction, the asymptotic neutron flux distribution in the $z$-direction decays exponentially according to $\exp (-\gamma z)$, where $\gamma$ is a spatial decay constant in the $z$-direction. The $\gamma$ is also a fundamental mode eigenvalue of the $\gamma$-mode transport equation (Yamamoto, 2012):

$$
\boldsymbol{L} \phi_{e}(\boldsymbol{r}, \boldsymbol{\Omega}, E)-\gamma \xi \phi_{e}(\boldsymbol{r}, \boldsymbol{\Omega}, E)=\frac{1}{k_{e}} \boldsymbol{F} \phi_{e}(\boldsymbol{r}, \boldsymbol{\Omega}, E),
$$

where $\xi=$ the direction cosine of $\Omega$ with the $z$-axis, and $\phi_{e}=$ the fundamental mode eigenfunction of the $\gamma$-eigenvalue mode equation. $\boldsymbol{F}$ is the same as Eq. (3) but $\chi$ and $v$ are for the sums of the prompt and delayed neutrons. Similarly, the second term on the left-hand side of Eq. (6) is taken into account by

$$
W_{j+1}=W_{j} \cdot \exp \left(\gamma \xi_{j} s_{j}\right),
$$

where $\xi_{j}=\xi$ of the $j$ th flight path. The remaining procedure is the same as that for the $k$ - $\alpha$ method. In the $k$ - $\alpha$ method, the weight of a particle always increases due to Eq. (4) when it moves. On the other hand, in the $k-\gamma$ method, the weight of a particle increases when it moves upward (i.e., $\xi_{j}>0$ ), and the weight decreases when it moves downward (i.e., $\xi_{j}<0$ ). After the convergence of the $k-\gamma$ iteration is achieved, the net current across the horizontal plane eventually is directed towards the upward direction. 


\section{Sensitivity analysis methods of $\alpha$ - and $\gamma$-eigenvalues}

\subsection{Sensitivity analysis method of $\alpha$-eigenvalue}

3 The Monte Carlo methods for the sensitivity analyses of the $k_{\text {eff }}$-eigenvalue that have been

4 developed thus far cannot be straightforwardly applied to the $\alpha$-eigenvalue in the $k$ - $\alpha$ method

5 because the $\alpha$-eigenvalue is not a true eigenvalue. Yamamoto and Sakamoto (2019) introduced the "time source" method to obtain the $\alpha$-eigenvalue for the sensitivity analyses of the $\alpha$-eigenvalue. The details of the "time source" method are presented in Shim et al. $(2014,2015)$ and Yamamoto and Sakamoto (2019). The equation to be solved for the "time source method" is

$$
\boldsymbol{L} \phi_{p}(\boldsymbol{r}, \boldsymbol{\Omega}, E)-\boldsymbol{F}_{\boldsymbol{p}} \phi(\boldsymbol{r}, \boldsymbol{\Omega}, E)=\alpha \frac{1}{v(E)} \phi_{p}(\boldsymbol{r}, \boldsymbol{\Omega}, E),
$$

9 which is the same as Eq. (1) except that $k_{p}$ is not included. The source in this calculation mode is $\phi_{p}(\boldsymbol{r}, \boldsymbol{\Omega}, E) / v(E)$ on the right-hand side instead of the fission source. The power iteration method is used to solve this equation by updating the source distribution in each cycle in the same way as with $k_{e f f}$-eigenvalue problems. The sources for the next cycle are determined at each collision point as follows:

$$
n=\operatorname{Int}\left[\alpha \cdot \frac{w}{v(E) \Sigma_{t}(\boldsymbol{r}, E)}+\eta\right]
$$

where $n=$ the number of sources at the collision point, $w=$ the weight of the colliding particle, and $\eta=$ uniform pseudo random number from $(0,1)$. Int $[x]$ denotes the largest integer not exceeding $x$. The $\alpha$ in Eq. (9) is introduced in order to keep the number of source particles almost constant throughout the cycles, and usually the $\alpha$-eigenvalue that is obtained in the previous cycle is used for it. However, a difficulty arises if the subcritical system contains a void region because no source can be defined by Eq. (9) in the void region. A workaround employed by Yamamoto and Sakamoto (2019) is to assign a virtual total cross-section to the void region. This method is similar to the Woodcock delta tracking method (Woodcock, 1965). A free flight distance $s$ in the void region is determined by $s=-\ell n \eta / \Sigma_{t v}$, where $\Sigma_{t v}$ is the virtual total cross-section. If a virtual collision occurs within the void region, the number of source particles is determined at the collision point by using $\Sigma_{t v}$ in Eq. (9). Then, the particle keeps flying without changing the weight and the direction as if it had undergone no reaction in the void region. At the end of each 
1 cycle, the $\alpha$-eigenvalue is calculated using the collision estimator as

$$
\alpha_{m}=\frac{S_{m}}{\sum_{j} \sum_{i} w_{i j} v\left(E_{i j}\right)^{-1} \Sigma_{t}\left(\boldsymbol{r}_{i j}, E_{i j}\right)^{-1}},
$$

2 where $i$ and $j$ denote the $i$ th collision and $j$ th source particles, respectively; $m=$ the cycle number; and $S_{m}=$ the sum of the source particle weight in the $m$ th cycle. The $\alpha$-eigenvalue can also be calculated using the track length estimator.

Yamamoto and Sakamoto (2019) used the first-order differential operator sampling (DOS) method (Rief, 1984; McKinney and Iverson, 1996; Raskach, 2009) to calculate the sensitivity coefficient of the $\alpha$-eigenvalue with respect to nuclear data $x$. The first derivative of the $\alpha$-eigenvalue with the DOS method is calculated for each history by

$$
\frac{\partial \alpha}{\partial x}=-\alpha^{2} \sum_{i} \frac{1}{v\left(E_{i}\right) \Sigma_{t}\left(\boldsymbol{r}_{i}, E_{i}\right)} w_{i} W_{x, i}
$$

9 where $i$ denotes the $i$ th collision of the history, $w_{i}$ is the particle weight at the $i$ th collision, and the summation is carried out over all collisions during the history. $W_{x, i}$ is the sum of the first-derivatives of the transport and collision kernels in the Neumann series solution. The explicit form of $W_{x, i}$ is given in Yamamoto and Sakamoto (2019) and will be presented later in this paper. The change of $x$ perturbs the distribution of the time source, thereby affecting the sensitivity coefficients. Eq. (11) does not include the perturbed source effect. A DOS method to estimate the perturbed source effect was already developed for the sensitivity coefficients of the $k_{\text {eff }}$-eigenvalue (Nagaya and Mori, 2005; Nagaya and Mori, 2011; Kiedrowski, 2017; Yamamoto, 2018). The perturbed source effect for the sensitivity coefficient of the $\alpha$-eigenvalue can be calculated with

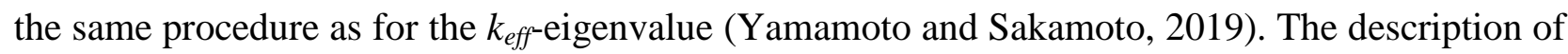
the perturbed source effect is omitted in this paper because it is not essentially related to the novelty of this paper. Details on how to calculate the perturbed source effect can be found in the references (Nagaya and Mori, 2005; Nagaya and Mori, 2011; Kiedrowski, 2017; Yamamoto, 2018).

\subsection{Difficulty in sensitivity analysis method of $\gamma$-eigenvalue}

If a sensitivity analysis method similar to the $\alpha$-eigenvalue that was presented in Sec. 3.1 was 
1 applied to the $\gamma$-eigenvalue, the source term would be given by the second term on the left-hand

side of Eq. (6). The equation to be solved is rewritten from Eq. (6) as

$$
\boldsymbol{L} \phi_{e}(\boldsymbol{r}, \boldsymbol{\Omega}, E)-\boldsymbol{F} \phi_{e}(\boldsymbol{r}, \boldsymbol{\Omega}, E)=\gamma \xi \phi_{e}(\boldsymbol{r}, \boldsymbol{\Omega}, E) .
$$

3 As in Eq. (9), the number of sources would be determined at each collision point:

$$
n=\operatorname{Int}\left[\gamma \cdot \frac{|\xi w|}{\Sigma_{t}(\boldsymbol{r}, E)}+\eta\right] \text {. }
$$

4 As described at the end of Sec.2.2, the directional cosine $\xi$ can be positive and negative, 5 depending on the particle direction. Thus, the positive and negative sources are produced by Eq. 6 (13). In such a case, the cancellation of the positive and negative sources needs to be performed at 7 the end of each cycle. Without the cancellation, the number of positive and negative sources keeps 8 growing, eventually resulting in divergence after several cycle iterations. The most common 9 technique for the cancellation is the "binning procedure". In the "binning procedure", the whole region is divided into a large number of small bins where positive and negative weights are summed up and cancelled (Booth, 2003; Yamamoto, 2009; Yamamoto, 2011; Zhang et al., 2016; Yamamoto and Sakamoto, 2018). Although the authors attempted to apply the "binning procedure" to $\gamma$-eigenvalue calculations, the procedure was unable to succeed in suppressing the growing positive and negative sources. According to (Mancusi and Zoia, 2018), chaotic behaviors that resulted in a series of periodic-doubling bifurcation were observed in buckling search calculations. The chaotic behavior might be related to the failure of the $\gamma$-eigenvalue mode calculations of this paper. Although regularization techniques could be attempted to prevent such a chaotic behavior, this issue is out of the scope of this paper. Consequently, the authors abandoned the attempt as a method to perform the sensitivity analysis of the $\gamma$-eigenvalue. In the next section, a newly developed sensitivity analysis method for $\alpha$ - and $\gamma$-eigenvalues is presented.

\section{Two-step method for sensitivity analyses of $\alpha$ - and $\gamma$-eigenvalues}

\subsection{Two-step sensitivity analysis method of $\alpha$-eigenvalue}

In this section, we devise a new algorithm for the sensitivity analyses of $\alpha$ - and $\gamma$-eigenvalues. For this purpose, the $k-\alpha$ or $k-\gamma$ iteration method are revisited. The sensitivity coefficient of the $\alpha$-eigenvalue with respect to nuclear data $x$ based on the linear perturbation theory is 


$$
S_{\alpha, x}=\frac{x}{\alpha} \frac{d \alpha}{d x}=\frac{-\left\langle\boldsymbol{\Phi}_{\mathrm{p}}^{*} x \frac{d \boldsymbol{F}_{p}}{d x} \boldsymbol{\Phi}_{\mathrm{p}}\right\rangle-\left\langle\boldsymbol{\Phi}_{\mathrm{p}}^{*} x \frac{d \boldsymbol{S}}{d x} \boldsymbol{\Phi}_{\mathrm{p}}\right\rangle+\left\langle\boldsymbol{\Phi}_{\mathrm{p}}^{*} x \frac{d \Sigma_{t}}{d x} \boldsymbol{\Phi}_{\mathrm{p}}\right\rangle}{\alpha\left\langle\boldsymbol{\Phi}_{\mathrm{p}}^{*} \boldsymbol{V}^{-1} \boldsymbol{\Phi}_{\mathrm{p}}\right\rangle},
$$

where $\boldsymbol{\Phi}_{\mathrm{p}}$ and $\boldsymbol{\Phi}_{\mathrm{p}}^{*}$ are the forward flux and the adjoint flux of $\alpha$-eigenvalue mode, respectively;

2\langle\rangle$=$ the integration over all the phase space; $\boldsymbol{F}_{p}=$ the production operator; $\boldsymbol{S}=$ the scattering 3 operator; and $\boldsymbol{V}^{-\mathbf{1}}=$ the inverse of neutron velocity. The formula in Eq. (14) is almost the same as 4 the one in (Endo and Yamamoto, 2018). Eq. (14) can be rearranged to yield the following:

$$
S_{\alpha, x}=\frac{x}{\alpha} \frac{d \alpha}{d x}=\frac{S_{k, x}^{p}}{S_{k, \alpha}^{p}}
$$

5 where

$$
S_{k, x}^{p} \equiv \frac{x}{k_{p}} \frac{d k_{p}}{d x}=\frac{-\left\langle\boldsymbol{\Phi}_{\mathrm{p}}^{*} x \frac{d \boldsymbol{F}_{p}}{d x} \boldsymbol{\Phi}_{\mathrm{p}}\right\rangle-\left\langle\boldsymbol{\Phi}_{\mathrm{p}}^{*} x \frac{d \boldsymbol{S}}{d x} \boldsymbol{\Phi}_{\mathrm{p}}\right\rangle+\left\langle\boldsymbol{\Phi}_{\mathrm{p}}^{*} x \frac{d \Sigma_{t}}{d x} \boldsymbol{\Phi}_{\mathrm{p}}\right\rangle}{\left\langle\boldsymbol{\Phi}_{\mathrm{p}}^{*} \boldsymbol{F}_{p} \boldsymbol{\Phi}_{\mathrm{p}}\right\rangle},
$$

6 and

$$
S_{k, \alpha}^{p} \equiv \frac{\alpha}{k_{p}} \frac{d k_{p}}{d \alpha}=\frac{\alpha\left\langle\boldsymbol{\Phi}_{\mathrm{p}}^{*} \boldsymbol{V}^{-1} \boldsymbol{\Phi}_{\mathrm{p}}\right\rangle}{\left\langle\boldsymbol{\Phi}_{\mathrm{p}}^{*} \boldsymbol{F}_{p} \boldsymbol{\Phi}_{\mathrm{p}}\right\rangle} .
$$

$7 \quad k_{p}$ does not explicitly appear in the right-hand side of both Eqs. (16) and (17) because $k_{P}=1$ in the $\alpha$-eigenvalue mode solution.

The sensitivity coefficient $S_{k, x}^{p}$ (i.e., Eq. (16)) can be calculated using the $k$ - $\alpha$ iteration method in the same manner as the Monte Carlo method for the $k_{\text {eff }}$-eigenvalue sensitivity coefficients. The sensitivity coefficient $S_{k, \alpha}^{p}$ (i.e., Eq. (17)) is the product of the prompt neutron generation time and the $\alpha$-eigenvalue. Thus, the Monte Carlo methods for calculating a prompt neutron generation time (e.g., Kiedrowski et al., 2011; Leppänen et al., 2014; Terranova and Zoia, 2017) can be used for calculating $S_{k, \alpha}^{p}$. The sensitivity coefficients $S_{k, x}^{p}$ and $S_{k, \alpha}^{p}$ can be calculated with several Monte Carlo methods. The generalized iteration fission probability (IFP) method (Terranova and Zoia, 2017; Jinaphanh and Zoia, 2019) is usable for this purpose. The generalized IFP can be extended to the $\alpha$-eigenvalue problem from the standard $k_{\text {eff }}$-eigenvalue problem.

This paper focuses on applying the differential operator sampling (DOS) method to calculate the sensitivity coefficients $S_{k, x}^{p}$ and $S_{k, \alpha}^{p}$. The DOS method for the sensitivity coefficients of the $k_{\text {eff }}$-eigenvalue and its memory reduction technique using the superhistory method (Brissenden and 
1 Garlick, 1986) were developed in (Yamamoto, 2018). The DOS method for the sensitivity

1

coefficients of the $k_{p}$-eigenvalue is almost the same as in (Yamamoto, 2018) except that the $k$ - $\alpha$ iteration is performed to obtain the $\alpha$-eigenvalue according to Eq. (1) instead of the $k_{\text {eff }}$-eigenvalue.

4 The procedure of the DOS method is briefly described here. Refer to (Yamamoto, 2018) for a 5 complete description of the DOS method.

6 The sensitivity coefficient of the $k_{p}$-eigenvalue with respect to nuclear data $x$ for the $m$ th particle history is given by

$$
S_{k, x, m}^{p}=\frac{x}{k_{p}} \frac{\partial}{\partial x} k_{p, m}=\frac{x}{k_{p}} \sum_{i} \frac{v_{p} \Sigma_{f, i}}{\Sigma_{t, i}} w_{i} W_{f, i},
$$

8 where $i$ denotes the $i$ th collision within the $m$ th history, $w_{i}$ is the particle weight at the $i$ th 9 collision, and the summation is carried out at every collision during the $m$ th history. $W_{f, i}$ is the weighting coefficient at the $i$ th collision, and it is defined as

$$
W_{f, i}=\frac{1}{v_{p} \Sigma_{f, i}} \frac{\partial}{\partial x} v_{p} \Sigma_{f, i}+\sum_{l=1}^{i-1} \frac{1}{\Sigma_{s, l}} \frac{\partial}{\partial x} \Sigma_{s, l}+\sum_{l=1}^{i-1} \frac{1}{f_{l}} \frac{\partial}{\partial x} f_{l}-\sum_{k} s_{k} \frac{\partial}{\partial x} \Sigma_{t, k},
$$

where $f_{l}$ is the angular and energy distribution of a scattered neutron. The second term on the right-hand side of Eq. (19) represents the sum of $1 / \Sigma_{s, l} \cdot \partial \Sigma_{s, l} / \partial x$ until the (i-1)th collision, where $\Sigma_{s, l}$ is the macroscopic scattering cross-section for the $l$ th scattering. The fourth term on the right-hand side of Eq. (19) denotes the sum of $s_{k} \cdot \partial \Sigma_{t, k} / \partial x$ in the $k$ th flight until the $i$ th collision, where $s_{k}$ is the flight distance of the $k$ th flight. $W_{f, i}$ is explicitly represented for some macroscopic cross-sections $x$ as follows:

$$
W_{f, i}=\left\{\begin{array}{c}
-\sum_{k} s_{k}, \quad x=\Sigma_{c}, \quad(20) \\
\sum_{l=1}^{i-1} \frac{1}{\Sigma_{s, l}}-\sum_{k} s_{k}, \quad x=\Sigma_{s},(21) \\
\frac{1}{\Sigma_{f, i}}-\sum_{k} s_{k}, \quad x=\Sigma_{f},(22) \\
1, \quad x=\chi_{p}, \quad(23) \\
\frac{1}{v_{p}}, \quad x=v_{p} .
\end{array}\right.
$$

17 Eq. (23) represents the weighting coefficient for the sensitivity with respect to the fission spectrum. 
1 In this paper, the sensitivity coefficient with respect to the fission spectrum is an unconstrained

1

one (Nagaya et al., 2009). Eq. (18) does not take into account the perturbation of the fission source distribution caused by the change of the nuclear data. The perturbation has a significant effect on the sensitivity coefficients. The formulation of the source perturbation effect is complicated and not essentially related to the objective of this paper. Presenting the detailed formulation for the source perturbation effect is omitted in this paper, and it can be found in (Nagaya and Mori, 2005; Kiedrowski, 2017; Yamamoto, 2018). After finishing all the histories in a cycle, the sensitivity coefficients that are calculated with Eq. (18) plus the source perturbation effect are averaged over all particle histories in the cycle, thus yielding the result of the cycle. Then, we proceed to the next cycle.

Next, the formulation of $S_{k, \alpha}^{p}$ for the DOS method is derived. A very similar DOS method was derived for the sensitivity of the $k_{\text {eff }}$-eigenvalue with respect to a complex-valued buckling (Yamamoto and Sakamoto, 2018). When a particle moves from position $\boldsymbol{r}$ to the collision point $\boldsymbol{r}^{\prime}$, the transport kernel in an $\alpha$-eigenvalue calculation is given by

$$
T\left(\boldsymbol{r} \rightarrow \boldsymbol{r}^{\prime}\right)=\Sigma_{t} \exp \left(-\Sigma_{t} s\right) \exp \left(\frac{\alpha}{v(E)} s\right),
$$

where $s=\left|\boldsymbol{r}^{\prime}-\boldsymbol{r}\right|$. In the normal Monte Carlo calculations, $\exp (\alpha s / v(E))$ in Eq. (25) is not included in the transport kernel. In the Monte Carlo calculation for the $\alpha$-eigenvalue, this term has to be multiplied because the particle weight changes during its flight, as given in Eq. (4). The weighting coefficient of the transport kernel with respect to $\alpha$, which is to be scored in each flight path, is

$$
\frac{1}{T\left(\boldsymbol{r} \rightarrow \boldsymbol{r}^{\prime}\right)} \cdot \frac{\partial}{\partial \alpha} T\left(\boldsymbol{r} \rightarrow \boldsymbol{r}^{\prime}\right)=\frac{s}{v(E)}
$$

Eq. (26) is scored for each flight path until the particle is discarded. This scoring is carried out throughout the whole system, including the void regions. The sensitivity coefficient of the $k_{p}$-eigenvalue with respect to $\alpha$ for the $m$ th particle history is given by

$$
S_{k, \alpha, m}^{p}=\frac{\alpha}{k_{p}} \frac{\partial}{\partial \alpha} k_{p, m}=\frac{\alpha}{k_{p}} \sum_{i} \frac{v_{p} \Sigma_{f, i}}{\Sigma_{t, i}} w_{i} T_{i},
$$

where $w_{i}=$ the particle weight of the $i$ th collision. The summation for $i$ is carried out at every 
1 collision point during the $m$ th history. The score $T_{i}$ at the $i$ th collision is

$$
T_{i}=\sum_{k} \frac{s_{k}}{v_{k}(E)}
$$

2 where the subscript $k$ denotes the $k$ th flight path and the summation is carried out for every flight 3 path until the $i$ th collision.

4 Eqs. (18) and (27) do not include the perturbed source effect. How to calculate this effect is presented in (Yamamoto, 2018) and (Yamamoto and Sakamoto, 2019). Thus, it is omitted in this paper.

\subsection{Two-step sensitivity analysis method of $\gamma$-eigenvalue}

A Monte Carlo algorithm for the sensitivity analysis of the $\gamma$-eigenvalue is straightforwardly derived according to the procedure in Sec. 4.1. The sensitivity coefficient of the $\gamma$-eigenvalue with respect to nuclear data $x$ based on the linear perturbation theory is

$$
S_{\gamma, x}=\frac{x}{\gamma} \frac{d \gamma}{d x}=\frac{-\left\langle\boldsymbol{\Phi}_{\mathrm{e}}^{*} x \frac{d \boldsymbol{F}}{d x} \boldsymbol{\Phi}_{\mathrm{e}}\right\rangle-\left\langle\boldsymbol{\Phi}_{\mathrm{e}}^{*} x \frac{d \boldsymbol{S}}{d x} \boldsymbol{\Phi}_{\mathrm{e}}\right\rangle+\left\langle\boldsymbol{\Phi}_{\mathrm{e}}^{*} x \frac{d \Sigma_{t}}{d x} \boldsymbol{\Phi}_{\mathrm{e}}\right\rangle}{\gamma\left\langle\boldsymbol{\Phi}_{\mathrm{e}}^{*} \xi \boldsymbol{\Phi}_{\mathrm{e}}\right\rangle},
$$

12 where $\boldsymbol{\Phi}_{\mathrm{e}}$ and $\boldsymbol{\Phi}_{\mathrm{e}}^{*}$ are the forward flux and the adjoint flux of $\gamma$-eigenvalue mode, respectively. In the same manner as in Sec. 4.1, Eq. (29) can be rearranged to yield the following:

14 where

$$
S_{\gamma, x}=\frac{x}{\gamma} \frac{d \gamma}{d x}=\frac{S_{k, x}^{e}}{S_{k, \gamma}^{e}}
$$

$$
S_{k, x}^{e} \equiv \frac{x}{k_{e}} \frac{d k_{e}}{d x}=\frac{-\left\langle\boldsymbol{\Phi}_{\mathrm{e}}^{*} x \frac{d \boldsymbol{F}}{d x} \boldsymbol{\Phi}_{\mathrm{e}}\right\rangle-\left\langle\boldsymbol{\Phi}_{\mathrm{e}}^{*} x \frac{d \boldsymbol{S}}{d x} \boldsymbol{\Phi}_{\mathrm{e}}\right\rangle+\left\langle\boldsymbol{\Phi}_{\mathrm{e}}^{*} x \frac{d \Sigma_{t}}{d x} \boldsymbol{\Phi}_{\mathrm{e}}\right\rangle}{\left\langle\boldsymbol{\Phi}_{\mathrm{e}}^{*} \boldsymbol{F} \boldsymbol{\Phi}_{\mathrm{e}}\right\rangle},
$$

and

$$
S_{k, \gamma}^{e} \equiv \frac{\gamma}{k_{e}} \frac{d k_{e}}{d \gamma}=\frac{\gamma\left\langle\boldsymbol{\Phi}_{\mathrm{e}}^{*} \xi \boldsymbol{\Phi}_{\mathrm{e}}\right\rangle}{\left\langle\boldsymbol{\Phi}_{\mathrm{e}}^{*} \boldsymbol{F} \boldsymbol{\Phi}_{\mathrm{e}}\right\rangle} .
$$

The sensitivity coefficient $S_{k, x}^{e}$ in Eq. (30) can be calculated in the same manner as for $S_{k, x}^{p}$ in Sec. 4.1 during the course of the $\gamma$-mode eigenvalue calculation. While $S_{k, x}^{p}$ is obtained by the $\alpha$-eigenvalue mode calculation, $S_{k, x}^{e}$ is obtained by the $\gamma$-eigenvalue mode calculation. Thus, $S_{k, x}^{p}$ is not equal to $S_{k, x}^{e}$, even for the same subcritical system.

Next, the formulation of $S_{k, \gamma}^{e}$ for the DOS method is derived. The transport kernel in the $\gamma$-eigenvalue calculation is given by

$$
T\left(\boldsymbol{r} \rightarrow \boldsymbol{r}^{\prime}\right)=\Sigma_{t} \exp \left(-\Sigma_{t} s\right) \exp (\gamma \xi s) .
$$


1 Thus, the weighting coefficient of the transport kernel with respect to $\gamma$ is

$$
\frac{1}{T\left(\boldsymbol{r} \rightarrow \boldsymbol{r}^{\prime}\right)} \cdot \frac{\partial}{\partial \gamma} T\left(\boldsymbol{r} \rightarrow \boldsymbol{r}^{\prime}\right)=\xi s .
$$

2 The sensitivity coefficient of the $k_{e}$-eigenvalue with respect to $\gamma$ for the $m$ th particle history is given by

$$
S_{k, \gamma, m}=\frac{\gamma}{k_{e}} \frac{\partial}{\partial \gamma} k_{e, m}=\frac{\gamma}{k_{e}} \sum_{i} \frac{v \Sigma_{f, i}}{\Sigma_{t, i}} w_{i} T_{i} .
$$

4 The score $T_{i}$ at the $i$ th collision is

$$
T_{i}=\sum_{k} \xi_{k} s_{k}
$$

5 where the subscript $k$ denotes the $k$ th flight path and the summation is carried out for every flight 6 path until the $i$ th collision.

\section{5. Numerical tests of the two-step method for sensitivity coefficients}

$9 \quad 5.1$ Two-step sensitivity analyses of $\alpha$-eigenvalue

The two-step sensitivity calculation method for the $\alpha$-eigenvalue using the DOS method is tested in this section. The geometry of the test problem is shown in Fig. 1 (the water-hole problem). The geometry is a two-dimensional rectangular shape where a light-water region is surrounded by a homogenized light-water moderated $\mathrm{UO}_{2}$ fuel rod array. Throughout this paper, the calculations are performed with three-energy group cross-sections and the scatterings are isotropic. Table 1 shows the group constants for the two materials. The sensitivity coefficients are calculated with respect to the macroscopic cross-sections (the capture, fission, and scattering cross-sections) and the fission spectrum in the $\mathrm{UO}_{2}$ region. A test Monte Carlo program is used for the numerical tests. The $k_{\text {eff }}$ and $\alpha$-eigenvalues of this subcritical system are $k_{\text {eff }}=0.84561 \pm$ 0.00004 and $\alpha=3823.8 \pm 0.2 \mathrm{~s}^{-1}$, respectively. The reference calculations for the sensitivity coefficients are performed with the discrete ordinates transport code DANTSYS (Alcouffe et al., 1995) using the same group constants. The three-energy group forward and adjoint angular fluxes of the $\alpha$-eigenvalue mode are calculated with the angular quadrature order 8 . Using the angular fluxes, the sensitivity coefficients are calculated according to Eq. (14) using a post-processing program developed by the authors. 
shows the perturbed source effect of the sensitivity coefficient of $k_{\text {eff }}$ with respect to the 1 st group capture cross-section as a function of the number of iterations. The results of the two-step method agree well with DANTSYS and the time source method within $0.4 \%$. The two-step method is much faster than the time source method. The figure of merit $(=1 /$ (square of standard deviation)/(cpu time)) of the two-step method is 17 times larger than that of the time source method.

[Fig. 2][Table 2][Table 3][Table 4][Table 5]

Another numerical test is performed for a two-dimensional graphite-reflected plutonium metal system. The configuration is shown in Fig. 3 (the Pu metal problem). Table 6 shows the group constants for the two materials. This problem is a small fast system where a large portion of neutrons are lost by leakage. The $k_{\text {eff }}$ and $\alpha$-eigenvalues of the Pu metal problem are $k_{\text {eff }}=0.92241$ \pm 0.00004 and $\alpha=244367 \pm 11 \mathrm{~s}^{-1}$, respectively. The ray effect in this system is significant, which makes the $\alpha$-eigenvalue (and possibly the sensitivity coefficients) sensitive to the angular quadrature order. Thus, the deterministic calculations using DANTSYS are not necessarily accurate and are omitted. The verification is performed by comparing the two-step method with the time source method. The sensitivity coefficients that are calculated with the time source method and the two-step method are compared in Table 7 (capture), Table 8 (fission), Table 9 (scattering), and Table 10 (fission spectrum (unconstrained)). The results of the two Monte Carlo methods are in good agreement with each other within $0.2 \%$ except for one case (fission spectrum 
[Fig. 3][Table 6][Table 7][Table 8][Table 9][Table 10]

A test problem showing that the new method performs properly for a subcritical system containing a void region is presented in Appendix.

\subsection{Two-step sensitivity analyses of $\gamma$-eigenvalue}

The sensitivity analyses of the $\gamma$-eigenvalue are performed for the two test problems in Sec. 5.1. The $\gamma$-eigenvalue of the first example (water-hole problem) is $\gamma=0.099972 \pm 0.000005 \mathrm{~cm}^{-1}$ ( $k_{\text {eff }}$ is the same as in Sec. 5.1). No currently available deterministic transport code is capable of performing $\gamma$-eigenvalue mode calculations. Thus, the reference solutions of the first-derivative of $\gamma$ with respect to a cross-section are approximately obtained from the two-independent Monte Carlo calculations for $\gamma$-eigenvalues as

$$
\frac{d \gamma}{d \Sigma} \approx \frac{\gamma_{p}-\gamma_{0}}{\Sigma_{p}-\Sigma_{0}}
$$

where the subscripts $p$ and $O$ denote the perturbed and unperturbed quantities, respectively. The sensitivity coefficients that are calculated with the two-step method are compared with those with the two-independent runs in Table 11 (capture), Table 12 (fission), and Table 13 (scattering). The sensitivity coefficient with respect to the fission spectrum is omitted because the two-independent runs cannot be performed for unconstrained sensitivity coefficients. It is difficult to draw a significant conclusion regarding the validity of the two-step method for $\gamma$-eigenvalue because the reference solutions are not exact, and furthermore, they entail relatively large statistical uncertainties. However, the results of the two-step method agree with the reference solutions within two standard deviations, which supports the validity of the proposed new method.

[Table 11][Table 12][Table 13]

The $\gamma$-eigenvalue of another example (the Pu metal problem) is $\gamma=0.075933 \pm 0.000002 \mathrm{~cm}^{-1}$. The sensitivity coefficients of the Pu metal problem are shown in Table 14 (capture) and Table 15 (fission). This fast subcritical system has very minor sensitivities to the scattering cross-sections. The results of the scattering cross-sections are omitted because accurate reference solutions can hardly be obtained from two independent runs. Again, the results of the two-step method agree 
1 well with the reference solutions within two standard deviations. The results in this section

1

suggest that the sensitivity coefficients of the $\gamma$-eigenvalue could be calculated correctly by using the proposed two-step method.

5 6. Conclusions

6 The authors recently developed a Monte Carlo sensitivity analysis method for the $\alpha$-eigenvalue without using the $k$ - $\alpha$ algorithm. However, the method is found to be inapplicable to the sensitivity analyses of the $\gamma$-eigenvalue (the spatial decay constant in an exponential experiment) because the positive and negative sources are generated in the $\gamma$-eigenvalue mode and the converged source distribution cannot be attained despite introducing the cancellation technique.

The sensitivity coefficient of the $\alpha$-eigenvalue, $S_{\alpha, x}$, is expressed differently using the ratio of two sensitivity coefficients as $S_{k, x}^{p} / S_{k, \alpha}^{p}$. Both sensitivity coefficients can be obtained by the $k$ - $\alpha$ algorithm using the differential operator sampling (DOS) method. In the same manner, the sensitivity coefficient of the $\gamma$-eigenvalue can be obtained by the $k-\gamma$ algorithm. This newly developed method is dubbed as the "two-step method" in this paper. The perturbed source effect and its memory reduction technique of the DOS method were already developed and these are also available for the purpose of this paper. Unlike the time source method, the two-step method can be applicable to critical or supercritical systems. The verification of the new method is performed by comparing the results for the three-energy group problems to the solutions of the deterministic transport method or to the approximate results that are obtained from the direct perturbations of the cross-sections.

The algorithm that is presented in this paper can be straightforwardly expanded to the sensitivity analyses of the $\alpha$ - and $\gamma$-eigenvalues in the continuous energy Monte Carlo. The sensitivity analysis of the effective delayed neutron fraction can be performed with the differential operator sampling method, which will be done as a future work. 


\section{Appendix}

The time source method and the two-step method for the sensitivity analysis of the $\alpha$-eigenvalue are tested for a subcritical system containing a void region. The geometry is shown in Fig. A1, which is very similar to Fig. 1. The cross-sections listed in Table 1 are used for the fuel region. The $k_{\text {eff }}$ and $\alpha$-eigenvalues of this subcritical system are $k_{e f f}=0.88386 \pm 0.00006$ and $\alpha=$ $3575.8 \pm 0.3 \mathrm{~s}^{-1}$, respectively. To avoid the redundancy, only the results for the 1st group are shown in Table A1, where the sensitivity coefficients calculated with DANTSYS, the time source method, and the two-step method are compared. The results of the discrete ordinates method are slightly biased due to the ray effect for the void-containing test problem. Thus, the results of DANTSYS do not exactly agree with the Monte Carlo methods. The newly proposed two-step method agree with the time source method within the two standard deviations, which suggests that the new method works properly for void-containing systems.

[Fig. A1][Table A1]

\section{References}

Alcouffe, R.E., Baker, R.S., Brinkley, F.W., Marr, D.R., O’Dell, R.D., Walters, W.F., 1995. DANTSYS: A diffusion accelerated neutral particle transport code system, LA-12969-M.

Aufiero, M., Bidaud, A., Hursin, M., Leppänen, J., Palmiotti, G., Pelloni, S., Rubiolo, P., 2015. A collision history-based approach to sensitivity/perturbation calculations in the continuous energy Monte Carlo code SERPENT, Ann. Nucl. Energy 84, 245-258.

Booth, T., 2003. Computing the higher $k$-eigenfunctions by Monte Carlo power iteration: a conjecture. Nucl. Eng. Sci. 143, 291-300.

Brissenden, R.J., Garlick, A.R., 1986. Biases in the estimation of $k_{\text {eff }}$ and its error by Monte Carlo methods. Ann. Nucl. Energy 113, 63-83.

Brockway, D., Soran, P., Whalen, P., 1985. Monte Carlo $\alpha$ calculation, Tech. Rep. LA-UR-85-1224, Los Alamos National Laboratory.

Endo, T., Yamamoto, A., 2018. Sensitivity analysis of prompt neutron decay constant using perturbation theory. J. Nucl. Sci. Technol. 55 (11), 1245-1254. 
1 Favorite, J. A., 2018. SENSMG: first-order sensitivities of neutron reaction rates, reaction-rate

2 ratios, leakage, $k_{\text {eff }}$, and $\alpha$ using PARTISN. Nucl. Eng. Sci. 192, 80-114.

Hayakawa, C.K., Spanier, J., Venugopalan, V., 2014. Comparative analysis of discrete and 4 continuous absorption weighting estimators used in Monte Carlo simulations of radiative $5 \quad$ transport in turbid media. J. Opt. Soc. Am. A 31(2), 301-311.

6 Jinaphanh, A., Leclaire, N., Cochet, B., 2016. Continuous-energy sensitivity coefficients in the MORET code, Nucl. Sci. Eng. 184, 53-68.

Jinaphanh, A., Zoia, A., 2019. Private Communication.

Kiedrowski, B.C., Brown, F.B., Wilson, P.P.H., 2011. Adjoint-weighted tallies for $k$-eigenvalue calculations with continuous-energy Monte Carlo. Nucl. Sci. Eng. 168, 226-241.

Kiedrowski, B.C., Brown, F.B., 2013. Adjoint-based $k$-eigenvalue sensitivity coefficients to nuclear data using continuous-energy Monte Carlo. Nucl. Sci. Eng. 174, 227-244.

Kiedrowski, B.C., 2017. Review of early 21st-century Monte Carlo perturbation and sensitivity techniques for $k$-eigenvalue radiation transport calculations. Nucl. Eng. Sci. 185, 426-444.

Leppänen, J., Aufiero, M., Fridman, E., Rachamin, R., van der Marck, S., 2014. Calculation of effective point kinetics parameters in the Serpent 2 Monte Carlo code. Ann. Nucl. Energy 65, $272-279$.

Mancusi, D. Zoia, A., 2018. Chaos in eigenvalue search methods. Ann. Nucl. Energy 112, $354-363$.

McKinney, G. W., Iverson, J. L., 1996. Verification of the Monte Carlo differential operator technique for MCNP, Los Alamos National Laboratory, LA-13098.

Nayaga, Y., Kodeli, I., Chiba, G., Ishikawa, M., 2009. Evaluation of sensitivity coefficients of effective multiplication factor with respect to prompt fission neutron spectrum. Nucl. Instrum. Meth. Phys. Res. A 603, 485-490.

Nagaya, Y., Mori, T., 2005. Impact of perturbed fission source on the effective multiplication factor in Monte Carlo perturbation calculations, J. Nucl. Sci. Technol., 42, 428-441.

Nagaya, Y., Mori, T., 2011. Estimation of sample reactivity worth with differential operator 
Perfetti, C. M., 2012. Advanced Monte Carlo methods for eigenvalue sensitivity coefficient calculations, Doctoral dissertation, University of Michigan.

Perfetti, C. M., Rearden, B. T., 2016. Development of a generalized perturbation theory method for sensitivity analysis using continuous-energy Monte Carlo methods, Nucl. Sci. Eng. 182, $354-368$.

Qiu, Y., Liang, J., Wang, K., Yu, J., 2015. New strategies of sensitivity analysis capabilities in continuous-energy Monte Carlo code RMC. Ann. Nucl. Energy 81, 50-61.

Qiu, Y., Aufiero, M., Wang, K., Fratoni, M., 2016a. Development of sensitivity analysis capabilities of generalized responses to nuclear data in Monte Carlo code RMC, Ann. Nucl. Energy 97, 142-152.

Qiu, Y., Shang, X., Tang, X., Liang, J., Wang, K., 2016b. Computing eigenvalue sensitivity coefficients to nuclear data by adjoint superhistory method and adjoint Wielandt method implemented in RMC code, Ann. Nucl. Energy 87, 228-241.

Raskach, K. F., 2009. An improvement of the Monte Carlo generalized differential operator method by taking into account first- and second-order perturbations of fission source. Nucl. Sci. Eng. 162, 158-166.

Rearden, B.T., 2004. Perturbation theory eigenvalue sensitivity analysis with Monte Carlo techniques. Nucl. Sci. Eng. 146, 367-382.

Rief, H., 1984. Generalized Monte Carlo perturbation algorithms for correlated sampling and a second-order Taylor series approach. Ann. Nucl. Energy 9, 455-476.

Shim, H.J., Kim, C.H., 2011. Adjoint sensitivity and uncertainty analyses in Monte Carlo forward calculations. J. Nucl. Sci. Technol. 48(12), 1453-1461.

Shim, H. J., Jeong, B.K., Kang, S.M., Kim, C.H., 2014. Monte Carlo alpha iteration algorithm for a prompt neutron decay constant calculation. Trans. Am. Nucl. Soc. 111, 725-726.

Shim, H.J., Jang, S.H., Kang, S.M., 2015. Monte Carlo alpha iteration algorithm for a subcritical system analysis. Sci. Technol. Nucl. Installations 2015, Article ID 859242. 
1 Suzaki, T., 1991. Subcriticality determination of low-enriched $\mathrm{UO}_{2}$ lattices in water by

2 exponential experiment. J. Nucl. Sci. Technol. 28(12) 1067-1077.

Suzaki, T., Kurosawa, M., Hirose, H., Yamamoto, T., Nakajima, K., Kanaizuka, F., Kobayashi, I., Kaneko, T., 1995. Exponential experiments of PWR spent fuel assemblies for acquiring subcriticality benchmarks usable in burnup credit applications," Proc. Intl. Conf. on Nucl. Criticality Safety, September 17-21, 1995, Albuquerque, New Mexico.

Terranova, N., Zoia, A., 2017. Generalized iterated fission probability for Monte Carlo eigenvalue calculations, Ann. Nucl. Energy 108, 57-66.

Terranova, N., Mancusi, D., Zoia, A., 2018. New perturbation and sensitivity capabilities in TRIPOLI-4 ${ }^{\circledR}$, Ann. Nucl. Energy 121, 335-349.

Woodcock, E. R., 1965. Techniques used in the GEM code for Monte Carlo neutronics calculations in reactors and other systems of complex geometry, Argonne National Laboratory, ANL-7050.

Yamamoto, T., Miyoshi, Y., Tonoike, K., Okamoto, H., Ida, T., Aoki, S., 2003. Effect of higher-harmonic flux in exponential experiment for subcriticality measurement. J. Nucl. Sci. Techonol. 40(2), 77-83.

Yamamoto, T., Miyoshi, Y., 2003. An algorithm of $\alpha$ - and $\gamma$-mode eigenvalue calculations by Monte Carlo method. Proc. 7th Int. Conf. on Nuclear Criticality Safety (ICNC '03), JAERI-Conf 2003-019, Japan Atomic Energy Research Institute, Tokai, Japan, October 2003.

Yamamoto, T., 2009. Convergence of the second eigenfunction in Monte Carlo power iteration. Ann. Nucl. Energy 36, 7-14.

Yamamoto, T., 2011. Higher order $\alpha$ mode eigenvalue calculation by Monte Carlo power iteration. Prog. Nucl. Sci. Technol. 2, 826-835.

Yamamoto, T., 2012. Monte Carlo algorithm for buckling search and neutron leakage-corrected calculations. Ann. Nucl. Energy 47, 14-20.

Yamamoto, T., 2018. Eigenvalue sensitivity analysis capabilities with the differential operator method in the superhistory Monte Carlo method. Ann. Nucl. Energy 112, 150-157. 
1 Yamamoto, T., Sakamoto, H., 2018. Monte Carlo method for solving a B1 equation with 2 complex-valued buckling in asymmetric geometries and generation of directional diffusion 3 coefficients. Ann. Nucl. Energy 122, 37-46.

4 Yamamoto, T., Sakamoto, H., 2019. A Monte Carlo technique for sensitivity analysis of 5 alpha-eigenvalue with the differential operator sampling method. Ann. Nucl. Energy 127, $6 \quad 178-187$.

7 Zhang, P., Lee, H., Lee. D., 2016. A general solution strategy of modified power method for $8 \quad$ higher mode solutions. J. Compt. Phys. 305, 387-402.

9 Zoia, A., Brun, E., Malvagi, F., 2014. Alpha eigenvalue calculations with TRIPOLI-4. Ann. Nucl. 10 Energy 63, 276-284. 11 24 


\section{List of figures}

2 Fig. 1 Geometry of the test problem for sensitivity analyses (the water-hole problem).

3 Fig. 2 Convergence of the perturbed source effect as a function of the number of iterations $\left(S_{k, \Sigma}^{p}\right.$

4 of the 1st group capture cross-section).

5 Fig. 3 Geometry of the test problem for sensitivity analyses (the Pu metal problem). 


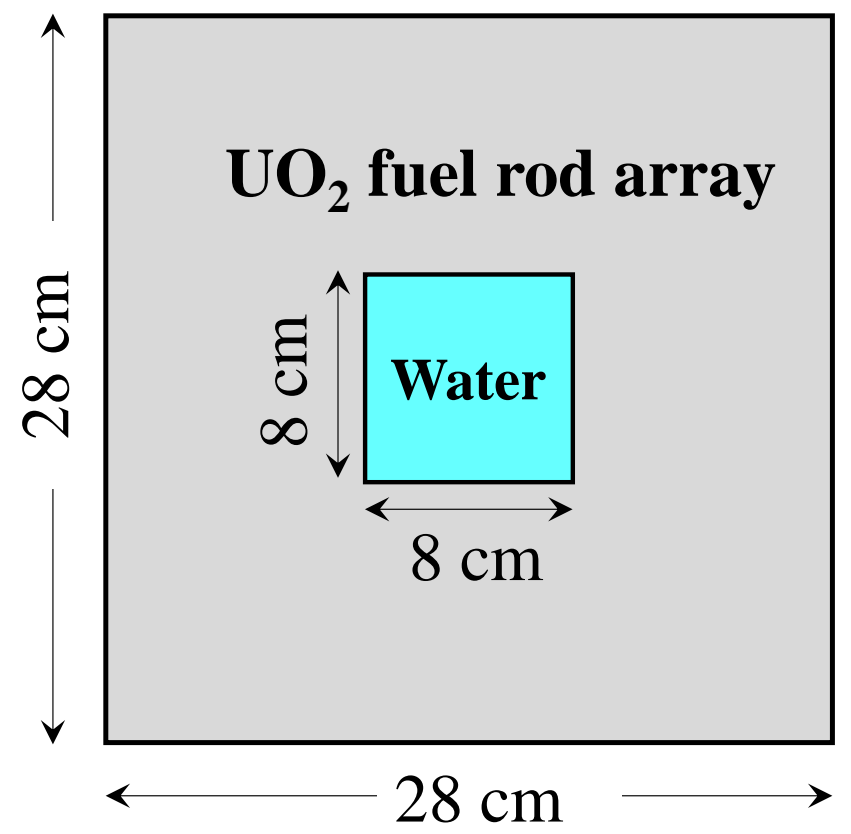

Fig. 1 Geometry of the test problem for sensitivity analyses (the water-hole problem). 


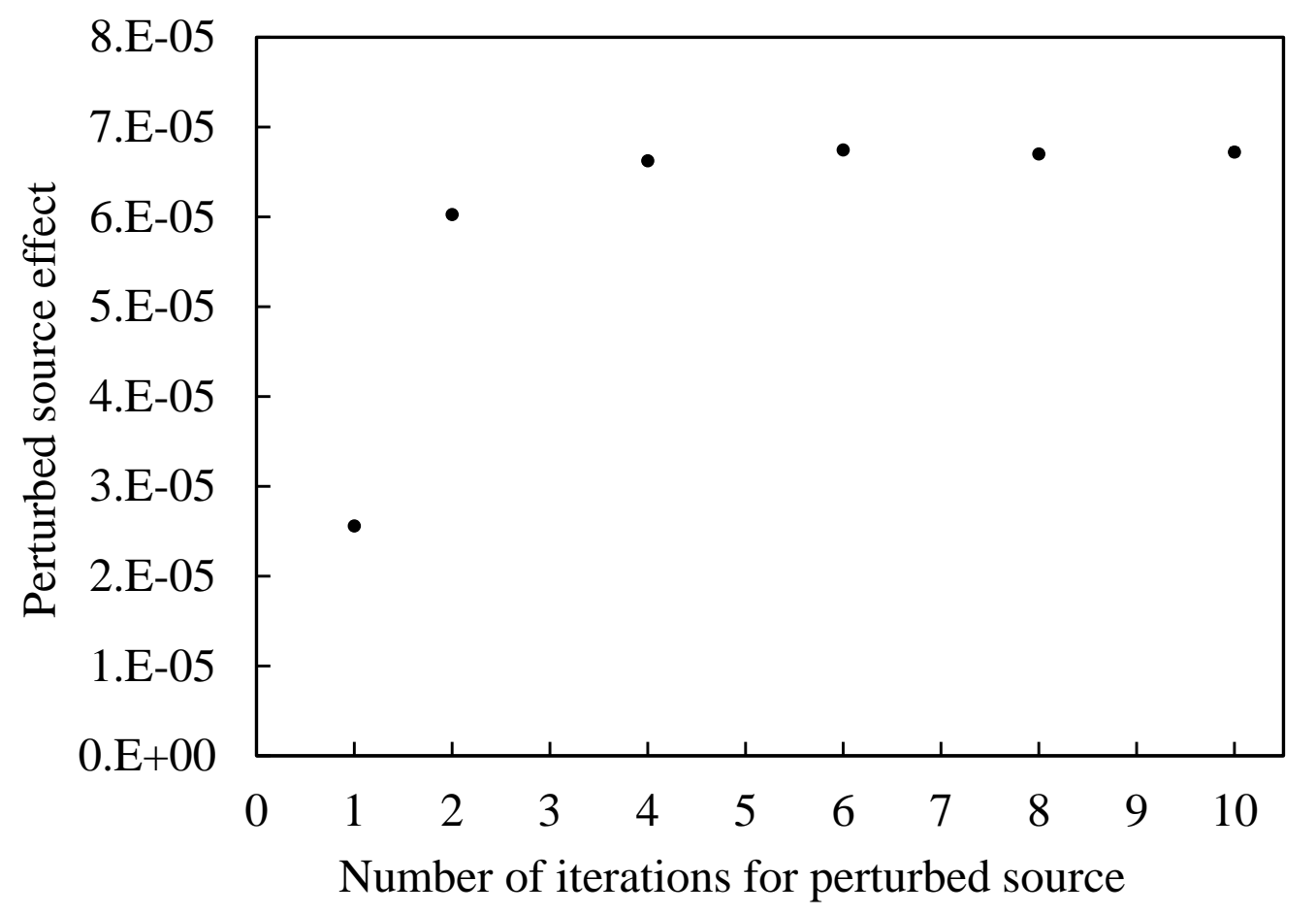

Fig. 2 Convergence of the perturbed source effect as a function of the number of iterations $\left(S_{k, \Sigma}^{p}\right.$ of the 1 st group capture cross-section). 


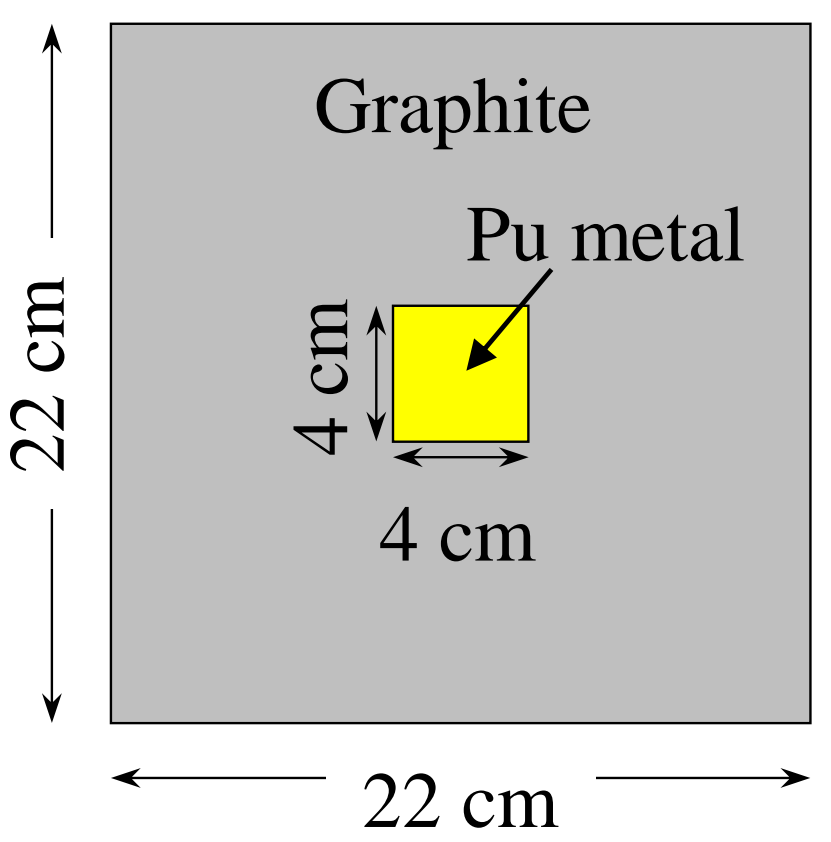

Fig. 3 Geometry of the test problem for sensitivity analyses (the Pu metal problem). 


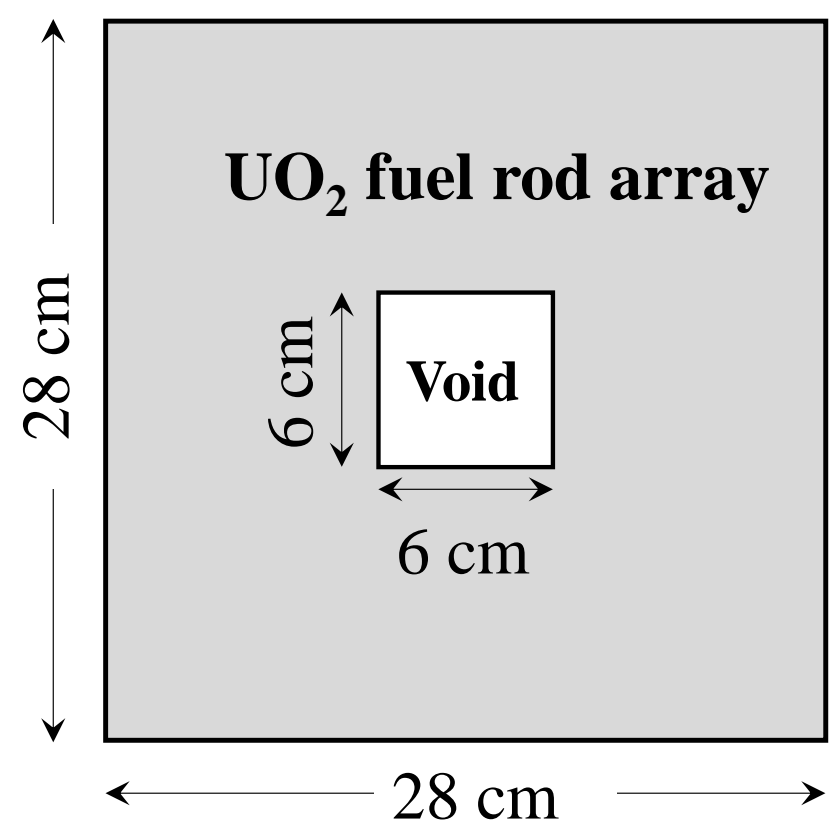

Fig. A1 Geometry of the test problem for sensitivity analyses (the void problem). 
Table 1 Three-group constants for $\mathrm{UO}_{2}$ fuel rod array and light water

\begin{tabular}{|c|c|c|c|}
\hline & & $\begin{array}{c}\mathrm{UO}_{2} \text { fuel rod } \\
\text { array }\end{array}$ & Light water \\
\hline \multirow{3}{*}{$\begin{array}{c}\text { Total cross } \\
\text { section }\left(\mathrm{cm}^{-1}\right)\end{array}$} & $\Sigma_{t 1}$ & 0.29829 & 0.33207 \\
\hline & $\Sigma_{t 2}$ & 0.83334 & 1.1265 \\
\hline & $\Sigma_{t 3}$ & 1.6389 & 2.7812 \\
\hline \multirow{3}{*}{$\begin{array}{l}\text { Fission cross } \\
\text { section }\left(\mathrm{cm}^{-1}\right)\end{array}$} & $\Sigma_{f 1}$ & 0.0030586 & - \\
\hline & $\Sigma_{f 2}$ & 0.0021579 & - \\
\hline & $\Sigma_{f 3}$ & 0.056928 & - \\
\hline \multirow{3}{*}{$\begin{array}{l}\text { Absorption } \\
\text { cross section } \\
\left(\mathrm{cm}^{-1}\right) \\
\end{array}$} & $\Sigma_{a 1}$ & 0.003385 & 0.00030500 \\
\hline & $\Sigma_{a 2}$ & 0.011895 & 0.00036990 \\
\hline & $\Sigma_{a 3}$ & 0.086180 & 0.0182500 \\
\hline \multirow{3}{*}{$\begin{array}{l}\text { Group transfer } \\
\text { cross section } \\
\left(\mathrm{cm}^{-1}\right)\end{array}$} & $\Sigma_{S}^{1 \rightarrow 2}$ & 0.073843 & 0.10464 \\
\hline & $\Sigma_{S}^{1 \rightarrow 3}$ & 0.0 & 0.0 \\
\hline & $\Sigma_{S}^{2 \rightarrow 3}$ & 0.043803 & 0.097961 \\
\hline $\begin{array}{c}\text { Neutrons per } \\
\text { fission }\end{array}$ & $v\left(v_{p}\right)$ & 2.4 & - \\
\hline \multirow{3}{*}{$\begin{array}{l}\text { Fission } \\
\text { spectrum }\end{array}$} & $\chi_{1}\left(\chi_{\mathrm{p} 1}\right)$ & 0.878198 & - \\
\hline & $\chi_{2}\left(\chi_{\mathrm{p} 2}\right)$ & 0.121802 & - \\
\hline & $\chi_{3}\left(\chi_{\mathrm{p} 3}\right)$ & 0 & - \\
\hline \multirow{3}{*}{$\begin{array}{l}\text { Neutron velocity } \\
\qquad(\mathrm{cm} / \mathrm{s})\end{array}$} & $v_{1}$ & \multicolumn{2}{|c|}{$1.66743 \times 10^{9}$} \\
\hline & $v_{2}$ & \multicolumn{2}{|c|}{$1.73734 \times 10^{7}$} \\
\hline & $v_{3}$ & \multicolumn{2}{|c|}{$3.46850 \times 10^{5}$} \\
\hline
\end{tabular}


Table $2 S_{\alpha, \Sigma}$ to the capture cross section (the water-hole problem).

\begin{tabular}{lccc}
\hline & 1 st Gr. & 2nd Gr. & 3rd Gr. \\
\hline DANTSYS & $1.1468 \times 10^{-2}$ & $5.4915 \times 10^{-1}$ & 1.5962 \\
\hline $\begin{array}{c}\text { Differential operator (MC) } \\
\text { Time source method }\end{array}$ & $1.1413 \times 10^{-2}$ & $5.4934 \times 10^{-1}$ & 1.5948 \\
& $\left(1.5 \times 10^{-5}\right)^{*}$ & $\left(7.2 \times 10^{-4}\right)$ & $\left(1.8 \times 10^{-3}\right)$ \\
\hline Two-step method & & \\
$S_{k, \alpha}^{p}$ & & $-2.3210 \times 10^{-1}\left(4 \times 10^{-5}\right)$ & \\
$S_{k, \Sigma}^{p}$ & $-2.6532 \times 10^{-3}$ & $-1.2707 \times 10^{-1}$ & $-3.7011 \times 10^{-1}$ \\
& $\left(6 \times 10^{-7}\right)$ & $\left(3 \times 10^{-5}\right)$ & $\left(7 \times 10^{-5}\right)$ \\
$S_{\alpha, \Sigma}\left(=S_{k, \Sigma}^{p} / S_{k, \alpha}^{p}\right)$ & $1.1431 \times 10^{-2}$ & $5.4749 \times 10^{-1}$ & 1.5946 \\
& $\left(3 \times 10^{-6}\right)$ & $\left(1.4 \times 10^{-4}\right)$ & $\left(4 \times 10^{-4}\right)$ \\
\hline MC/DANTSYS & 1.003 & 1.003 & 1.000 \\
\hline
\end{tabular}

*Absolute one standard deviation

Table $3 S_{\alpha, \Sigma}$ to the fission cross section (the water-hole problem).

\begin{tabular}{lccc}
\hline & 1 st Gr. & 2nd Gr. & 3rd Gr. \\
\hline DANTSYS & $-1.7234 \times 10^{-1}$ & $-1.2738 \times 10^{-1}$ & -1.4688 \\
\hline Differential operator (MC) & & & \\
Time source method & $-1.7145 \times 10^{-1}$ & $-1.2711 \times 10^{-1}$ & -1.4679 \\
& $\left(1.9 \times 10^{-4}\right)^{*}$ & $\left(5.4 \times 10^{-4}\right)$ & $\left(2.5 \times 10^{-3}\right)$ \\
\hline
\end{tabular}

Two-step method

$$
\begin{aligned}
& S_{k, \alpha}^{p} \\
& S_{k, \Sigma}^{p}
\end{aligned}
$$

$$
S_{\alpha, \Sigma}\left(=S_{k, \Sigma}^{p} / S_{k, \alpha}^{p}\right)
$$

$$
-2.3210 \times 10^{-1}\left(4 \times 10^{-5}\right)
$$

$3.9950 \times 10^{-2}$

$2.9486 \times 10^{-2}$

$3.4027 \times 10^{-1}$

$\left(4.0 \times 10^{-5}\right)$

$\left(3.8 \times 10^{-5}\right)$

$\left(1.1 \times 10^{-4}\right)$

$-1.7212 \times 10^{-1}$

$-1.2704 \times 10^{-1}$

$-1.4661$

MC/DANTSYS

$\left(1.8 \times 10^{-4}\right)$

$\left(1.6 \times 10^{-4}\right)$ $\left(6 \times 10^{-4}\right)$

\footnotetext{
* Absolute one standard deviation
} 
Table $4 S_{\alpha, \Sigma}$ to the scattering cross section (the water-hole problem).

\begin{tabular}{lcc}
\hline & 1 st Gr. & 2nd Gr. \\
\hline DANTSYS & -1.1903 & -1.4938 \\
\hline $\begin{array}{c}\text { Differential operator (MC) } \\
\text { Time source method }\end{array}$ & -1.1893 & -1.4978 \\
& $\left(3.4 \times 10^{-3}\right)^{*}$ & $\left(5.7 \times 10^{-3}\right)$ \\
\hline Two-step method & $-2.3210 \times 10^{-1}$ & $\left(4 \times 10^{-5}\right)$ \\
$S_{k, \alpha}^{p}$ & $2.7634 \times 10^{-1}$ & $3.4593 \times 10^{-1}$ \\
& $\left(2.6 \times 10^{-4}\right)$ & $\left(5.9 \times 10^{-4}\right)$ \\
$S_{\alpha, \Sigma}\left(=S_{k, \Sigma}^{p} / S_{k, \alpha}^{p}\right)$ & -1.1906 & -1.4904 \\
& $\left(1.1 \times 10^{-3}\right)$ & $\left(2.5 \times 10^{-3}\right)$ \\
\hline MC/DANTSYS & 1.000 & 0.998 \\
\hline
\end{tabular}

* Absolute one standard deviation

Table $5 S_{\alpha, \Sigma}$ to the fission spectrum (the water-hole problem).

\begin{tabular}{lcc}
\hline & $1 \mathrm{st} \mathrm{Gr}$ & 2nd Gr. \\
\hline DANTSYS & -4.3654 & $-7.3868 \times 10^{-1}$ \\
\hline Differential operator (MC) & & \\
Time source method & -4.3549 & $-7.3906 \times 10^{-1}$ \\
& $\left(5.2 \times 10^{-3}\right)^{*}$ & $\left(1.22 \times 10^{-3}\right)$ \\
\hline Two-step method & $-2.3210 \times 10^{-1}$ & $\left(4 \times 10^{-5}\right)$ \\
$S_{k, \alpha}^{p}$ & 1.0114 & $1.7125 \times 10^{-1}$ \\
$S_{k, \Sigma}^{p}$ & $\left(1.8 \times 10^{-3}\right)$ & $\left(7 \times 10^{-5}\right)$ \\
$S_{\alpha, \Sigma}\left(=S_{k, \Sigma}^{p} / S_{k, \alpha}^{p}\right)$ & -4.3577 & $-7.3782 \times 10^{-1}$ \\
& $\left(7.8 \times 10^{-3}\right)$ & $\left(3.2 \times 10^{-4}\right)$ \\
\hline MC/DANTSYS & 0.9982 & 0.9988 \\
\hline
\end{tabular}

* Absolute one standard deviation 
Table 6 Three-group constants for the plutonium metal and the graphite

\begin{tabular}{|c|c|c|c|}
\hline & & Plutonium metal & Graphite \\
\hline \multirow{3}{*}{$\begin{array}{l}\text { Total cross } \\
\text { section }\end{array}$} & $\Sigma_{t 1}\left(\mathrm{~cm}^{-1}\right)$ & 0.28573 & 0.21053 \\
\hline & $\Sigma_{t 2}\left(\mathrm{~cm}^{-1}\right)$ & 0.35423 & 0.45009 \\
\hline & $\Sigma_{t 3}\left(\mathrm{~cm}^{-1}\right)$ & 0.62448 & 0.53500 \\
\hline \multirow{3}{*}{$\begin{array}{l}\text { Production } \\
\text { cross section }\end{array}$} & $\Sigma_{f 1}\left(\mathrm{~cm}^{-1}\right)$ & 0.072424 & - \\
\hline & $\Sigma_{f 2}\left(\mathrm{~cm}^{-1}\right)$ & 0.052973 & - \\
\hline & $\Sigma_{f 3}\left(\mathrm{~cm}^{-1}\right)$ & 0.13267 & - \\
\hline \multirow{4}{*}{$\begin{array}{l}\text { Absorption } \\
\text { cross section }\end{array}$} & $\Sigma_{a 1}\left(\mathrm{~cm}^{-1}\right)$ & 0.073056 & 0.00013890 \\
\hline & $\Sigma_{a 2}\left(\mathrm{~cm}^{-1}\right)$ & 0.064640 & 0.0000017 \\
\hline & $\Sigma_{a 3}\left(\mathrm{~cm}^{-1}\right)$ & 0.022681 & 0.000021 \\
\hline & $\Sigma_{S}^{1 \rightarrow 2}\left(\mathrm{~cm}^{-1}\right)$ & 0.029374 & 0.029672 \\
\hline \multirow{2}{*}{$\begin{array}{l}\text { Group transfer } \\
\text { cross section }\end{array}$} & $\Sigma_{S}^{1 \rightarrow 3}\left(\mathrm{~cm}^{-1}\right)$ & 0 & 0 \\
\hline & $\Sigma_{S}^{2 \rightarrow 3}\left(\mathrm{~cm}^{-1}\right)$ & 0.00030767 & 0.015913 \\
\hline $\begin{array}{l}\text { Neutrons per } \\
\text { fission }\end{array}$ & $v\left(v_{p}\right)$ & 3.2 & - \\
\hline \multirow{3}{*}{$\begin{array}{l}\text { Fission } \\
\text { spectrum }\end{array}$} & $\chi_{1}\left(\chi_{\mathrm{p} 1}\right)$ & 0.774148 & - \\
\hline & $\chi_{2}\left(\chi_{\mathrm{p} 2}\right)$ & 0.22485 & - \\
\hline & $\chi_{3}\left(\chi_{\mathrm{p} 3}\right)$ & 0.001002 & - \\
\hline \multirow{3}{*}{$\begin{array}{c}\text { Neutron } \\
\text { velocity }(\mathrm{cm} / \mathrm{s})\end{array}$} & $v_{1}$ & \multicolumn{2}{|c|}{$1.91275 \times 10^{9}$} \\
\hline & $v_{2}$ & \multicolumn{2}{|c|}{$6.97560 \times 10^{8}$} \\
\hline & $v_{3}$ & \multicolumn{2}{|c|}{$1.68994 \times 10^{7}$} \\
\hline
\end{tabular}

Table $7 S_{\alpha, \Sigma}$ to the capture cross section (the Pu metal problem).

\begin{tabular}{cccc}
\hline & 1 st Gr. & 2nd Gr. & 3rd Gr. \\
\hline $\begin{array}{c}\text { Differential operator (MC) } \\
\text { Time source method } \\
\text { (reference) }\end{array}$ & $9.6011 \times 10^{-3}$ & $1.6637 \times 10^{-1}$ & $4.2247 \times 10^{-1}$ \\
\hline Two-step method & $\left(8.7 \times 10^{-6}\right)^{*}$ & $\left(1 \times 10^{-5}\right)$ & $\left(3.2 \times 10^{-4}\right)$ \\
$S_{k, \alpha}^{p}$ & \multicolumn{3}{c}{} \\
$S_{k, \Sigma}^{p}$ & $-1.5392 \times 10^{-1}\left(1.4 \times 10^{-4}\right)$ \\
& $-1.4795 \times 10^{-3}$ & $-2.5573 \times 10^{-2}$ & $-3.7011 \times 10^{-1}$ \\
$S_{\alpha, \Sigma}\left(=S_{k, \Sigma}^{p} / S_{k, \alpha}^{p}\right)$ & $\left(2 \times 10^{-7}\right)$ & $\left(6 \times 10^{-6}\right)$ & $\left(7 \times 10^{-5}\right)$ \\
& $9.6116 \times 10^{-3}$ & $1.6614 \times 10^{-1}$ & $4.2271 \times 10^{-1}$ \\
& $\left(8.8 \times 10^{-6}\right)$ & $\left(1.6 \times 10^{-4}\right)$ & $\left(4.4 \times 10^{-4}\right)$ \\
\hline (Two-step)/(reference) & 1.001 & 0.999 & 1.001 \\
\hline
\end{tabular}

* Absolute one standard deviation 
Table $8 S_{\alpha, \Sigma}$ to the fission cross section (the Pu metal problem).

\begin{tabular}{cccc}
\hline & 1 st Gr. & 2nd Gr. & 3rd Gr. \\
\hline $\begin{array}{c}\text { Differential operator (MC) } \\
\text { Time source method } \\
\text { (reference) }\end{array}$ & -2.6969 & -1.3722 & $-5.8808 \times 10^{-1}$ \\
\hline Two-step method & $\left(2.6 \times 10^{-3}\right)^{*}$ & $\left(1.4 \times 10^{-3}\right)$ & $\left(2.1 \times 10^{-4}\right)$ \\
$S_{k, \alpha}^{p}$ & & & \\
$S_{k, \Sigma}^{p}$ & & $-1.5392 \times 10^{-1}\left(1.4 \times 10^{-4}\right)$ & \\
& $4.1529 \times 10^{-1}$ & $2.1149 \times 10^{-1}$ & $9.0640 \times 10^{-2}$ \\
$S_{\alpha, \Sigma}\left(=S_{k, \Sigma}^{p} / S_{k, \alpha}^{p}\right)$ & $\left(6 \times 10^{-5}\right)$ & $\left(3 \times 10^{-5}\right)$ & $\left(6.9 \times 10^{-5}\right)$ \\
& -2.6980 & -1.3740 & $-5.8886 \times 10^{-1}$ \\
& $\left(2.5 \times 10^{-3}\right)$ & $\left(1.2 \times 10^{-3}\right)$ & $\left(7.0 \times 10^{-4}\right)$ \\
\hline (Two-step)/(reference) & 1.000 & 1.001 & 1.001 \\
\hline
\end{tabular}

* Absolute one standard deviation

Table $9 S_{\alpha, \Sigma}$ to the scattering cross section (the Pu metal problem).

\begin{tabular}{ccc}
\hline & 1 st Gr. & 2nd Gr. \\
\hline $\begin{array}{c}\text { Differential operator (MC) } \\
\text { Time source method } \\
\text { (reference) }\end{array}$ & $-2.2085 \times 10^{-1}$ & $-5.6971 \times 10^{-2}$ \\
\hline Two-step method & $\left(1.03 \times 10^{-3}\right)^{*}$ & $\left(7.65 \times 10^{-4}\right)$ \\
$S_{k, \alpha}^{p}$ & $-1.5392 \times 10^{-1}$ & $\left(1.4 \times 10^{-4}\right)$ \\
$S_{k, \Sigma}^{p}$ & $3.4291 \times 10^{-2}$ & $8.8336 \times 10^{-3}$ \\
$S_{\alpha, \Sigma}\left(=S_{k, \Sigma}^{p} / S_{k, \alpha}^{p}\right)$ & $\left(9.0 \times 10^{-5}\right)$ & $\left(9.45 \times 10^{-5}\right)$ \\
& $-2.2278 \times 10^{-1}$ & $-5.7390 \times 10^{-2}$ \\
\hline (Two-step)/(reference) & $\left(6.2 \times 10^{-4}\right)$ & $\left(6.16 \times 10^{-4}\right)$ \\
\hline
\end{tabular}

* Absolute one standard deviation 
Table $10 S_{\alpha, \Sigma}$ to the fission spectrum (the Pu metal problem).

\begin{tabular}{cccc}
\hline & 1 st Gr. & 2nd Gr. & 3rd Gr. \\
\hline $\begin{array}{c}\text { Differential operator (MC) } \\
\text { Time source method } \\
\text { (reference) }\end{array}$ & -5.3214 & -1.7099 & $-1.0964 \times 10^{-2}$ \\
\hline Two-step method & $\left(4.7 \times 10^{-3}\right)^{*}$ & $\left(1.6 \times 10^{-3}\right)$ & $\left(7 \times 10^{-6}\right)$ \\
$S_{k, \alpha}^{p}$ & & & \\
$S_{k, \Sigma}^{p}$ & & $-1.5392 \times 10^{-1}\left(1.4 \times 10^{-4}\right)$ & \\
& $8.1928 \times 10^{-1}$ & $2.6309 \times 10^{-1}$ & $1.6999 \times 10^{-3}$ \\
$S_{\alpha, \Sigma}\left(=S_{k, \Sigma}^{p} / S_{k, \alpha}^{p}\right)$ & $\left(7 \times 10^{-5}\right)$ & $\left(6 \times 10^{-5}\right)$ & $\left(5.3 \times 10^{-6}\right)$ \\
& -5.3226 & -1.7092 & $-1.1044 \times 10^{-2}$ \\
\hline (Two-step)/(reference) & $\left(4.9 \times 10^{-3}\right)$ & $\left(1.6 \times 10^{-3}\right)$ & $\left(3.6 \times 10^{-5}\right)$ \\
\hline
\end{tabular}

* Absolute one standard deviation

Table $11 S_{\gamma, \Sigma}$ to the capture cross section (the water-hole problem).

\begin{tabular}{lccc}
\hline & 1 st Gr. & 2nd Gr. & 3rd Gr. \\
\hline Two-independent runs & $7.535 \times 10^{-3}$ & $3.247 \times 10^{-1}$ & $7.618 \times 10^{-1}$ \\
(reference) & $\left(6.1 \times 10^{-5}\right)^{*}$ & $\left(1.8 \times 10^{-3}\right)$ & $\left(3.5 \times 10^{-3}\right)$ \\
\hline Two-step method & \multicolumn{3}{c}{} \\
\multicolumn{3}{c}{$S_{k, \gamma}^{e}$} & $-3.5678 \times 10^{-1}\left(1.3 \times 10^{-4}\right)$ \\
\multicolumn{1}{c}{$S_{k, \Sigma}^{e}$} & $-2.6490 \times 10^{-3}$ & $-1.1640 \times 10^{-1}$ & $-2.7428 \times 10^{-1}$ \\
& $\left(6 \times 10^{-7}\right)$ & $\left(2 \times 10^{-5}\right)$ & $\left(4 \times 10^{-5}\right)$ \\
$S_{\gamma, \Sigma}\left(=S_{k, \Sigma}^{e} / S_{k, \gamma}^{e}\right)$ & $7.425 \times 10^{-3}$ & $3.262 \times 10^{-1}$ & $7.687 \times 10^{-1}$ \\
& $\left(3 \times 10^{-6}\right)$ & $\left(2 \times 10^{-4}\right)$ & $\left(3 \times 10^{-4}\right)$ \\
\hline (Two-step)/(reference) & 0.985 & 1.005 & 1.009 \\
\hline
\end{tabular}

* Absolute one standard deviation 
Table $12 S_{\gamma, \Sigma}$ to the fission cross section (the water-hole problem).

\begin{tabular}{|c|c|c|c|}
\hline & 1st Gr. & 2nd Gr. & 3rd Gr. \\
\hline $\begin{array}{l}\text { Two-independent runs } \\
\text { (reference) }\end{array}$ & $\begin{array}{c}-1.039 \times 10^{-1} \\
\left(6 \times 10^{-4}\right)^{*}\end{array}$ & $\begin{array}{c}-8.676 \times 10^{-2} \\
\left(5.3 \times 10^{-4}\right)\end{array}$ & $\begin{array}{c}-9.738 \times 10^{-1} \\
\left(7.0 \times 10^{-3}\right)\end{array}$ \\
\hline \multicolumn{4}{|l|}{ Two-step method } \\
\hline$S_{k, \gamma}^{e}$ & \multicolumn{3}{|c|}{$-3.5678 \times 10^{-1}\left(1.3 \times 10^{-4}\right)$} \\
\hline$S_{k, \Sigma}^{e}$ & $\begin{array}{l}3.6857 \times 10^{-2} \\
\left(2.8 \times 10^{-5}\right)\end{array}$ & $\begin{array}{l}3.1157 \times 10^{-2} \\
\left(2.7 \times 10^{-5}\right)\end{array}$ & $\begin{array}{c}3.4753 \times 10^{-1} \\
\left(7 \times 10^{-5}\right)\end{array}$ \\
\hline$S_{\gamma, \Sigma}\left(=S_{k, \Sigma}^{e} / S_{k, \gamma}^{e}\right)$ & $\begin{array}{c}-1.0330 \times 10^{-1} \\
\left(9 \times 10^{-5}\right)\end{array}$ & $\begin{array}{c}-8.733 \times 10^{-2} \\
\left(8 \times 10^{-5}\right)\end{array}$ & $\begin{array}{c}-9.741 \times 10^{-1} \\
\left(4 \times 10^{-4}\right)\end{array}$ \\
\hline (Two-step)/(reference) & 0.994 & 1.007 & 1.000 \\
\hline
\end{tabular}

* Absolute one standard deviation

Table $13 S_{\gamma, \Sigma}$ to the scattering cross section (the water-hole problem).

\begin{tabular}{lcc}
\hline & 1 st Gr. & 2nd Gr. \\
\hline $\begin{array}{l}\text { Two-independent runs } \\
\text { (reference) }\end{array}$ & $-3.306 \times 10^{-1}$ & $-6.841 \times 10^{-1}$ \\
\hline Two-step method & $\left(2.1 \times 10^{-3}\right)^{*}$ & $\left(4.6 \times 10^{-3}\right)$ \\
$\qquad S_{k, \gamma}^{e}$ & & \\
$S_{k, \Sigma}^{e}$ & $-3.5678 \times 10^{-1}$ & $\left(1.3 \times 10^{-4}\right)$ \\
& $1.1863 \times 10^{-1}$ & $2.4645 \times 10^{-1}$ \\
$S_{\gamma, \Sigma}\left(=S_{k, \Sigma}^{e} / S_{k, \gamma}^{e}\right)$ & $\left(2.2 \times 10^{-4}\right)$ & $\left(3.9 \times 10^{-4}\right)$ \\
& $-3.3251 \times 10^{-1}$ & $-6.9077 \times 10^{-1}$ \\
& $\left(6.4 \times 10^{-4}\right)$ & $\left(1.14 \times 10^{-3}\right)$ \\
\hline (Two-step)/(reference) & 1.006 & 1.010 \\
\hline
\end{tabular}

* Absolute one standard deviation 
Table $14 S_{\gamma, \Sigma}$ to the capture cross section (the Pu metal problem).

\begin{tabular}{lccc}
\hline & 1 st Gr. & 2nd Gr. & 3rd Gr. \\
\hline Two-independent runs & $8.019 \times 10^{-3}$ & $1.224 \times 10^{-1}$ & $2.027 \times 10^{-1}$ \\
(reference) & $\left(2.1 \times 10^{-5}\right)^{*}$ & $\left(3 \times 10^{-4}\right)$ & $\left(1.6 \times 10^{-3}\right)$ \\
\hline Two-step method & & & \\
\multicolumn{1}{c}{$S_{k, \gamma}^{e}$} & & $-1.8358 \times 10^{-1}\left(7 \times 10^{-5}\right)$ & \\
$S_{k, \Sigma}^{e}$ & $-1.4694 \times 10^{-3}$ & $-2.2421 \times 10^{-2}$ & $-3.7156 \times 10^{-2}$ \\
& $\left(3 \times 10^{-7}\right)$ & $\left(6 \times 10^{-6}\right)$ & $\left(1.9 \times 10^{-5}\right)$ \\
$S_{\gamma, \Sigma}\left(=S_{k, \Sigma}^{e} / S_{k, \gamma}^{e}\right)$ & $8.004 \times 10^{-3}$ & $1.2212 \times 10^{-1}$ & $2.024 \times 10^{-1}$ \\
& $\left(3 \times 10^{-6}\right)$ & $\left(6 \times 10^{-5}\right)$ & $\left(1 \times 10^{-4}\right)$ \\
\hline (Two-step)/(reference) & 0.998 & 1.005 & 0.996 \\
\hline
\end{tabular}

* Absolute one standard deviation

Table $15 S_{\gamma, \Sigma}$ to the fission cross section (the Pu metal problem).

\begin{tabular}{lccc}
\hline & 1 st Gr. & 2nd Gr. & 3rd Gr. \\
\hline Two-independent runs & -2.143 & -1.181 & $-3.821 \times 10^{-1}$ \\
(reference) & $\left(1.1 \times 10^{-2}\right)^{*}$ & $\left(3 \times 10^{-4}\right)$ & $\left(1.1 \times 10^{-3}\right)$
\end{tabular}

Two-step method

$$
\begin{aligned}
& S_{k, \gamma}^{e} \\
& S_{k, \Sigma}^{e}
\end{aligned}
$$

$3.9023 \times 10^{-1}$

$$
-1.8358 \times 10^{-1}\left(7 \times 10^{-5}\right)
$$

$\left(6 \times 10^{-5}\right)$

$2.1761 \times 10^{-1}$

$6.9856 \times 10^{-2}$

$$
S_{\gamma, \Sigma}\left(=S_{k, \Sigma}^{e} / S_{k, \gamma}^{e}\right)
$$

$-2.1256$

$\left(5 \times 10^{-5}\right)$

$\left(3.1 \times 10^{-5}\right)$$$
\left(9 \times 10^{-4}\right)
$$

$-1.1853$

$-3.805 \times 10^{-1}$

\begin{tabular}{lccc} 
& $\left(9 \times 10^{-4}\right)$ & $\left(5 \times 10^{-4}\right)$ & $\left(2 \times 10^{-4}\right)$ \\
\hline (Two-step)/(reference) & 0.992 & 1.004 & 0.996 \\
\hline
\end{tabular}

* Absolute one standard deviation 
Table A1 $S_{\alpha, \Sigma}$ for the 1st group cross-sections (the void problem).

\begin{tabular}{lccc}
\hline & Capture & Fission & Scattering \\
\hline DANTSYS & $1.9533 \times 10^{-2}$ & $-2.9620 \times 10^{-1}$ & -2.1415 \\
\hline $\begin{array}{c}\text { Differential operator (MC) } \\
\text { Time source method } \\
\text { (reference) }\end{array}$ & $1.9241 \times 10^{-2}$ & $-2.9005 \times 10^{-1}$ & -2.1177 \\
\hline Two-step method & $\left(5.6 \times 10^{-5}\right)^{*}$ & $\left(1.03 \times 10^{-3}\right)$ & $\left(7.1 \times 10^{-3}\right)$ \\
$\qquad \begin{array}{c}S_{k, \alpha}^{p} \\
S_{k, \Sigma}^{p}\end{array}$ & $-1.5463 \times 10^{-1}\left(1.4 \times 10^{-4}\right)$ & \\
& $-2.9836 \times 10^{-3}$ & $4.5015 \times 10^{-2}$ & $3.2963 \times 10^{-1}$ \\
$S_{\alpha, \Sigma}\left(=S_{k, \Sigma}^{p} / S_{k, \alpha}^{p}\right)$ & $\left(6 \times 10^{-7}\right)$ & $\left(3.5 \times 10^{-5}\right)$ & $\left(2.3 \times 10^{-4}\right)$ \\
& $1.9296 \times 10^{-2}$ & $-2.9111 \times 10^{-1}$ & -2.1318 \\
& $\left(1.8 \times 10^{-5}\right)$ & $\left(3.5 \times 10^{-4}\right)$ & $\left(2.5 \times 10^{-3}\right)$ \\
\hline (Two-step)/(reference) & 1.003 & 1.004 & 1.007 \\
\hline
\end{tabular}

* Absolute one standard deviation 